\title{
Global undernutrition during gestation influences learning during adult life
}

\author{
Jason Landon, Michael Davison, Christian U. Krägeloh, Nichola M. Thompson, \\ Jennifer L. Miles, Mark H. Vickers, Mhoyra Fraser, And Bernhard H. Breier \\ University of Auckland, Auckland, New Zealand
}

\begin{abstract}
Intrauterine growth restriction can lead to significant long-term health consequences such as metabolic and cardiovascular disorders, but less is known about its effects on choice and behavioral adaptation in later life. Virgin Wistar rats were time mated and randomly assigned to receive either ad-libitum access to chow or $30 \%$ of that level of nutrition during pregnancy to generate growth-restricted offspring. At 60 days of age, 6 female offspring from each group were trained on concurrent variable-interval schedules. Sessions consisted of seven randomly arranged concurrent-schedule components, each with a different reinforcer ratio that varied from 27:1 to $1: 27$, and each component lasting for 10 reinforcer deliveries. Behavioral change across reinforcers in components, measured by sensitivity to reinforcement, was consistently lower for offspring of undernourished mothers, showing that their behavior was less adaptable to environmental change. These results provide direct experimental evidence for a link between prenatal environmental conditions and reduced behavioral adaptability_-learning — in later life.
\end{abstract}

Converging evidence from epidemiological and experimental studies has suggested a link between intrauterine growth restriction and an increased susceptibility to the development of metabolic and cardiovascular disorders later in life (Gluckman \& Hanson, 2004). Epidemiological data have also shown a positive correlation between birth weight and cognitive function until early adulthood (Richards, Hardy, Kuh, \& Wadsworth, 2001, 2002). As a consequence, interest has grown in the possibility of a relationship between prenatal events and later cognitive function. Because animal studies permit control over both pre- and postnatal conditions, they are preferable to epidemiological research in the investigation of this relationship.

A range of animal models of intrauterine growth restriction has been developed. These have often involved arranging protein malnutrition or decreasing the overall plane of nutrition during gestation. Morgane, Mokler, and Galler (2002) suggested three main ways through which nutritional and environmental factors might affect the central nervous system's capability and performance: (1) alteration of brain function so as to reduce learning, due to abnormalities of morphological, biochemical, and physiological characteristics; (2) impairment of developmental processes by decreasing exposure and responsiveness to enriching stimuli during critical periods; and (3) disruption of learning processes via adverse changes in "personality, emotionality, and behavior" (p. 482). It is clear that these pathways are not necessarily independent, and at present it would be very difficult to delineate them. Nonetheless, a fundamental question remains: What effect, if any, does prenatal nutritional restriction have on an animal's subsequent ability to learn and to adapt to its environment?

A number of studies have investigated the effects of early-life nutrition on behavior during adulthood, but the results have been mixed. Tonkiss, Galler, Formica, ShukittHale, and Timm (1990) found that rats with prenatal protein malnutrition showed impaired response acquisition on a differential reinforcement of low rate (DRL) schedule. Tonkiss, Shultz, and Galler (1994) reported no effect of prenatal malnutrition on performance in a Morris water maze; Tonkiss, Shultz, Shumsky, and Galler (1997) found a very small deficit in performance. Tonkiss and Galler (1990) found no differences in working memory performance as a result of prenatal protein malnutrition in a rewarded alternation task using a T-maze. However, in an operant version of the rewarded alternation task, they found that prenatally malnourished rats performed significantly better than controls at the longest delay interval. This result was in direct contrast to the researchers' hypothesis that prenatal protein malnutrition would impair working memory performance, and also suggested that there might be some conditions under which prenatally malnourished offspring could perform better than controls.

The research described above establishes, in principle, that prenatal events can have effects on later learning and behavior. However, a somewhat confused picture remains of the nature and magnitude of the effects-more complex and detailed operant procedures may simply be better at detecting some of the more subtle effects of prenatal events on later behavior.

M. Davison, m.davison@auckland.ac.nz 
In the present research, we used a concurrent-schedule procedure introduced and reported on extensively by Davison and colleagues (e.g., Davison \& Baum, 2000, 2002; Landon \& Davison, 2001; Landon, Davison, \& Elliffe, 2003). This procedure has been used to examine the speed of learning in pigeons under a range of variable environments. It is a variant of a two-alternative concurrent schedule with frequent and directionally unpredictable changes in the reinforcer ratio available from two response alternatives. Sessions are divided into seven components, each of which has a different arranged reinforcer ratio. As opposed to conventional steady-state concurrent-schedule procedures, in which each reinforcer ratio is arranged in a separate condition (usually over several weeks), the present procedure arranges a range of reinforcer-ratio variations in each session. Studies using this procedure have shown that adaptation to the frequent changes in the reinforcer ratio can be very fast, and the rate of adaptation is affected by environmental variables such as the range of variation and the overall reinforcer rate.

The repeated within-session exposures to a range of reinforcer ratios allows rate of learning to be measured using an analysis based on the generalized matching law (Baum, 1974). This analysis measures the linear relation between $\log$ response ratios (choice) and log reinforcer ratios between the response alternatives following each successive reinforcer delivery in components. This relation is known as "sensitivity to reinforcement" (Baum, 1974). At the start of a component, sensitivity to reinforcement is typically slightly negative (reflecting control by the previous-component reinforcer ratio) and increases with successive component reinforcers to values of 0.4 to 0.6. More detailed analyses have examined the effects of individual reinforcers and specific sequences of reinforcers on choice and have highlighted striking regularities in these effects.

The present study used this approach to examine the effects of an established model of intrauterine growth restriction in the rat (e.g., Vickers, Breier, Cutfield, Hofman, \& Gluckman, 2000; Woodall, Johnston, Breier, \& Gluckman, 1996) on behavioral adaptation and learning. The manipulation involved a reduction in the overall plane of nutrition during gestation. At birth, intrauterine growth restricted (IUGR) pups are cross-fostered onto ad-libitum-fed dams so that postnatal nutrition is standardized. This induces small offspring that develop a range of symptoms in later life, including obesity, increased systolic blood pressure, and increased fasting insulin levels (see Breier, Vickers, Ikenasio, Chan, \& Wong, 2001, for a review).

\section{METHOD}

\section{Subjects}

Virgin Wistar rats were time mated at $100 \pm 5$ days of age. All rats were kept in the same room with a constant temperature maintained at $25^{\circ} \mathrm{C}$ and a $12: 12$-h light:dark cycle. A rat estrous cycle monitor was used to assess the stage of estrous of the animals before introducing the male. After confirmation of mating, the rats were housed individually in standard rat cages containing wood shavings as bedding, with free access to water. Pregnant dams were randomly assigned to either a control group that continued to receive standard rat chow (Teklad 18\% Protein Diet, Oxon.) ad libitum throughout gestation or a group that received $30 \%$ of the control group intake throughout gestation (IUGR group; see, e.g., Vickers et al., 2000; Woodall et al., 1996). Food intake and maternal weights were recorded daily until the end of pregnancy. Mating of dams that were randomly designated to become IUGR mothers was delayed by 1 day, and the controlled food intake of these dams was calculated from the mean intake of the control dams on the same day following mating. After birth, pups were weighed, and litter size for both control and IUGR dams was adjusted to 8 pups per litter to ensure adequate and standardized nutrition until weaning. Pups from the control group remained with their biological mothers from birth until weaning. To prevent a carryover of prenatal undernutrition to the immediate neonatal period, IUGR pups from undernourished mothers were cross-fostered onto dams that received ad libitum feeding throughout pregnancy. Thus, IUGR offspring were provided with the same nutrient-rich environment after birth as were control offspring, and IUGR pups were cross-fostered onto control dams that had their offspring removed after birth. This experimental approach generates IUGR offspring of significantly reduced birth weight without affecting litter size (Vickers et al., 2000; Vickers, Ikenasio, \& Breier, 2001; Woodall et al., 1999; Woodall et al., 1996). For the present study, we used conditions identical to those reported by our laboratory for physiological, metabolic, and endocrine studies investigating the impact of maternal undernutrition during gestation on the biological phenotype of offspring. Twelve female offspring (6 control and 6 IUGR) were used for this study. Pairs of littermates were housed in standard plastic cages containing wood shavings as bedding with metal bar tops and with water freely available. In the control group, two of the three pairs of rats came from the same litter, and in the IUGR group, each of the pairs of rats was from a different litter. From weaning until 60 days of age, all offspring were provided with ad-libitum access to standard chow. Prior to commencing behavioral training, food access was restricted to $2 \mathrm{~h}$ per day. Behavioral testing sessions were conducted daily between 0900 and $1200 \mathrm{~h}$ and were followed by 2 -h access to food in the rats' home cages. All rats were exposed to a 12:12 light:dark cycle with lights on at 0600 and off at $1800 \mathrm{~h}$. This study was approved by the University of Auckland Animal Ethics Committee.

\section{Apparatus}

Ten standard operant chambers for rats (ENV-007, Med Associates) with the dimensions $305 \times 241 \times 292 \mathrm{~mm}$ were situated in sound- and light-attenuating cabinets. The left and right walls consisted of three aluminium channels into which modular components could be inserted. The chambers were fitted with two retractable response levers (ENV-112CM) and a centrally located pellet receptacle, on the right wall. The back wall and the door consisted of clear polycarbonate, and the floor consisted of metal bars above a removable tray filled with wood shavings. A houselight provided ambient light, and a fan provided ventilation and masked external noise. All experimental events were arranged remotely by a personal computer running MED-PC software. The computer recorded the time at which every event occurred in each experimental session.

\section{Procedure}

Pretraining. Leverpressing was autoshaped in 60-min daily sessions. According to a variable-time 60 -sec schedule, one of the two response levers was presented $(p=.5)$ for $20 \mathrm{sec}$, followed by retraction of the lever and delivery of a $45-\mathrm{mg}$ Noyes food pellet (Research Diets, New Brunswick, NJ). If, during the $20 \mathrm{sec}$, a lever response occurred, the lever was retracted immediately and a food pellet delivered. Once responses were recorded to least $95 \%$ of lever presentations in a session, leverpressing was considered shaped.

Following autoshaping, and prior to the commencement of Condition 1, further training was required. A training session began with the presentation of two response levers on which reinforcers were scheduled with equal probability. After 10 reinforcers, both response levers were retracted for $10 \mathrm{sec}$. Reinforcers were scheduled 
dependently (Stubbs \& Pliskoff, 1969), meaning that once a reinforcer was arranged at one alternative, no further reinforcers could be arranged until that reinforcer was delivered. A changeover delay (Herrnstein, 1961) was introduced such that no reinforcer could be obtained within a certain time interval after a change between response alternatives. Gradually across sessions, the changeover delay was increased to $2 \mathrm{sec}$ and the overall probability of reinforcement per second was reduced from 1 to .037 .

Experimental procedure. A two-lever concurrent-schedule procedure was used, and sessions began with the left and right levers being inserted into the chamber. Each session contained seven unsignaled components that arranged the following concurrent VI:VI reinforcer ratios: 1:27, 1:9, 1:3, 1:1, 3:1, 9:1, or 27:1 (Davison \& Baum, 2000). The overall VI schedules were arranged by querying a probability gate, set at different probability values in the three conditions of the experiment, every $1 \mathrm{sec}$ and allocating reinforcers to response alternatives according to the reinforcer ratio required for each component. Reinforcers were scheduled dependently, and a 2-sec changeover delay (Herrnstein, 1961) was in effect. Components lasted until 10 reinforcers had been delivered; the components were separated by a 10 -sec period during which both response levers were retracted and the schedules were stopped. After this 10 -sec period, the next component began with the levers again being inserted into the chamber.

In each session, the order of the seven components was random without replacement. Immediately prior to the beginning of each component, the computer controlling the experiment randomly selected the reinforcer ratio to be in effect from a list of the seven possible reinforcer ratios. In a 1:27 component, for example, the probability of an arranged reinforcer's being allocated was $1 / 28$ to the left alternative, and $27 / 28$ to the right alternative. Thus, a reinforcer was 27 times more likely to be arranged on the right alternative than on the left. In Conditions 1-3, the overall rates of reinforcement were $2.22,6.00$, and 1.50 per min, respectively, and these conditions were run for 54,51 , and 80 daily sessions, respectively. Data collection consisted of recording the times that every experimental event occurred. Sessions were conducted daily and ended in blackout following the completion of all seven components or after $45 \mathrm{~min}$ had elapsed, whichever occurred first. Following sessions, rats were given $2 \mathrm{~h}$ of free access to food in their home cages.

\section{RESULTS}

Figure 1 shows the mean body weights in grams for both groups as a function of age. Body weights of control offspring were significantly higher than those of IUGR offspring at Day $51(t$ test, $p<.01)$, and Days 195 and 257 ( $t$ test, both $p$ s $<.05$ ).

As in previous research using this procedure (Davison \& Baum, 2000; Landon \& Davison, 2001; Landon et al., 2003), data from the last 35 sessions of each condition were used in the analyses.

\section{Behavioral Adaptation}

The sensitivity-to-reinforcement parameter of the generalized matching law (Baum, 1974; see Davison \& McCarthy, 1988, for a review) provides a measure of the degree of behavioral adaptation to environmental change. In its logarithmic form, it is written as

$$
\log \left(\frac{B_{1}}{B_{\mathrm{r}}}\right)=a \log \left(\frac{R_{1}}{R_{\mathrm{r}}}\right)+\log c
$$

where $B$ is the number of responses emitted and $R$ the numbers of reinforcers obtained on the left and right alter-

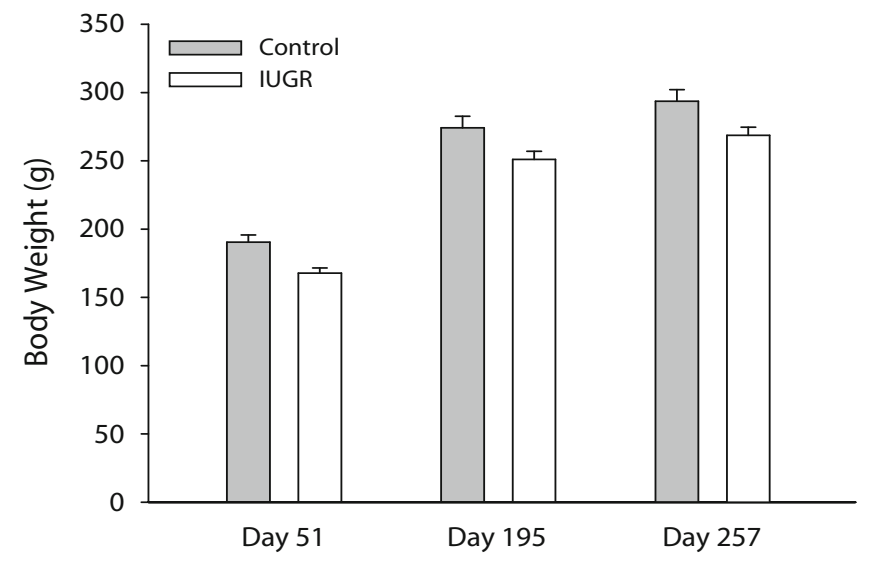

Figure 1. Mean group body weights in grams $(+S E M)$ for Days 51,195 , and 257 since birth.

natives (subscripts "l" and " $r$ "). $\log c$ is inherent key bias. The parameter $a$, sensitivity to reinforcement (Lobb \& Davison, 1975), describes the extent to which variations in the log reinforcer ratio change the log response ratio, and this parameter is thus a measure of degree of behavioral adaptation. The change of the value of $a$ across successive reinforcers in components is a measure of learning.

Linear-regression analyses for each subject estimated sensitivity to reinforcement separately after each successive component reinforcer, to investigate the extent to which preference adapted to the reinforcer ratio with every additional reinforcer delivery in components. For this analysis, we summed the number of responses $B_{1}$ and $B_{\mathrm{r}}$ emitted on each alternative in each component for the periods before the first reinforcer was obtained (Reinforcer 0 ), for the periods between the first and second reinforcers in a component (Reinforcer 1), and so on, until the 9th reinforcer. For the linear regression, $\log \left(R_{\mathrm{l}} / R_{\mathrm{r}}\right)$ was the obtained numbers of reinforcers in each component. The Optimizer function in Quattro Pro was used to find the value of sensitivity to reinforcement $a_{n}$ and $\log c$ that best described the changes in the $\log$ response ratio $B_{1 \mathrm{rn}} /$ $B_{\mathrm{rn}}$ as a function of variations in the component reinforcer ratio.

Figure 2 shows mean group sensitivity to reinforcement values, $a$, as a function of successive reinforcer deliveries in a component. Sensitivity values increased with successive reinforcer deliveries and appeared to approximate an asymptotic level by the end of components. When the overall reinforcer rate was increased to 6 reinforcers $/ \mathrm{min}$ in Condition 2, sensitivity values increased considerably for both groups (as reported by Davison \& Baum, 2000). When the reinforcer rate was reduced to 1.50 reinforcers/ min in Condition 3 , sensitivity values decreased, but not to levels lower than those obtained in Condition 1. Mean sensitivity values were $21 \%$ lower for IUGR offspring than for control offspring (repeated measures ANOVA, $F=$ 5.30, $p<.05, N=12$, with two between-subjects factors and nine within-subjects factors). For this comparison, sensitivity at Reinforcer 0 (before the first component reinforcer) was not included, because only sensitivity val- 

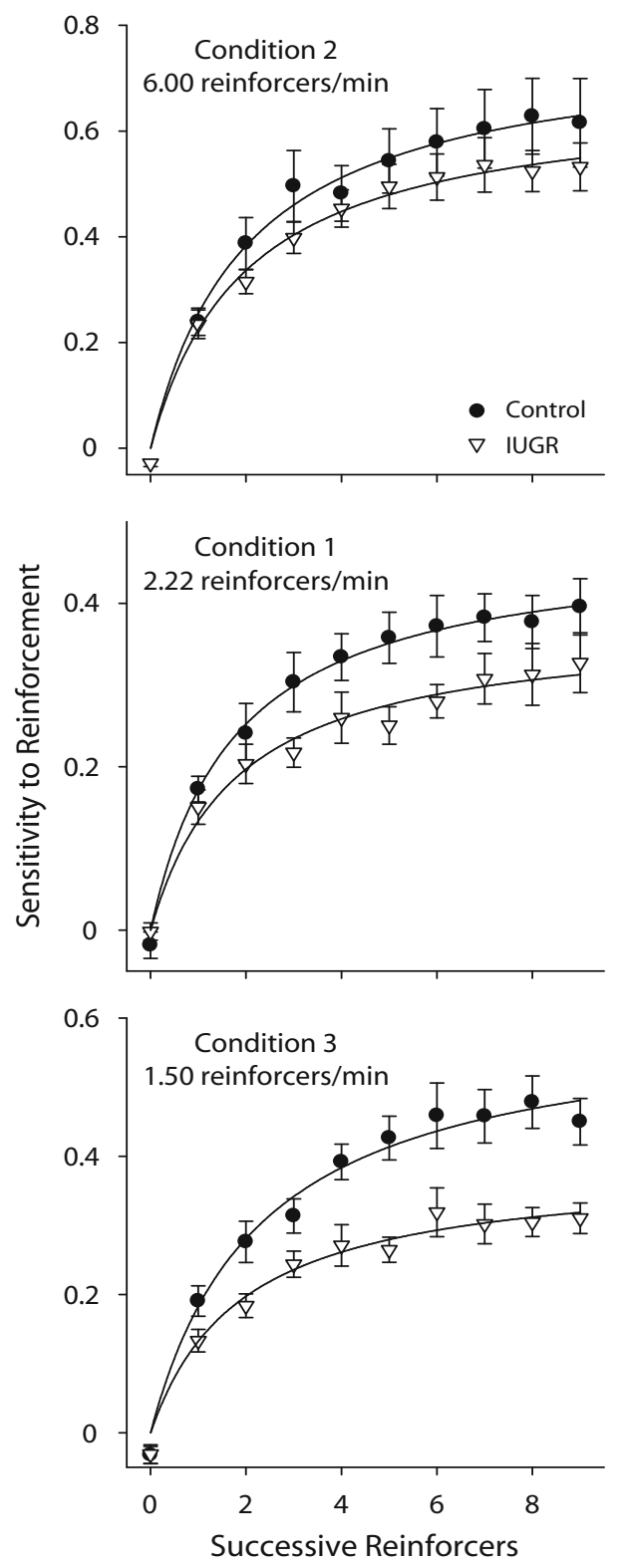

Figure 2. Mean sensitivity to reinforcement as a function of successive reinforcers for controls (filled circles) and intrauterine growth restricted (IUGR; unfilled triangles) offspring for Conditions 1-3. Error bars are $\pm 1 S E M$, and fitted curves are simple increasing hyperbolae to the group mean data.

ues after the delivery of at least one component reinforcer are meaningful measures of behavioral adaptation. There were no significant interactions between group effects and the effects of successive reinforcers or overall reinforcer rates. In addition to performing these analyses, we fitted the following hyperbolic growth curve to individual data:

$$
y=\frac{m x}{b+x} .
$$

The hyperbolic-growth-curve analysis confirmed the preceding results. The values of the asymptote, $m$, were significantly higher in the control group than in the IUGR group (repeated measures ANOVA, $F=5.94, p<.05, N=12$, with two between-subjects factors and nine within-subjects factors). There was no significant group difference in $b$, which measures the curvature of the hyperbola.

\section{Local Changes in Preference}

The following analyses investigated moment-to-moment preference after reinforcement (Davison \& Baum, 2002). The patterns of local changes in preference immediately after a reinforcer were termed "preference pulses" by Davison and Baum (2002) because there is typically a transitory shift in preference following a reinforcer delivery toward the alternative at which the reinforcer was obtained. This pulse is followed by a movement toward indifference, at least when the overall sessional numbers of reinforcers are the same on both alternatives (as in the present experiment; cf. Landon et al., 2003).

$\log$ (left/right) response ratios were calculated for each successive response after a reinforcer delivery. Previous research using this procedure has highlighted the particular importance of sequences of reinforcers obtained at the same alternative (Davison \& Baum, 2000, 2002; Landon \& Davison, 2001; Landon et al., 2003). In order to provide an overall measure of preference for the alternative at which the reinforcer was just obtained, $\log$ response ratios after right-alternative reinforcers were multiplied by -1 and then averaged with log response ratios after left-alternative reinforcers. Figure 3 shows representative examples of preference pulses from Condition 1 for IUGR and control offspring separately. From left to right, the five panels show preference pulses after successive sequences of reinforcers on the same alternative (i.e., L or R, LL or RR, LLL or RRR, LLLL or RRRR, and LLLLL or RRRRR) from the beginning of a component. Preference pulses were evident in the behavior of both IUGR and control groups: Both IUGR and control offspring were likely to continue responding at the alternative from which a reinforcer had just been obtained and were increasingly likely to stay at the reinforced alternative as successive same-alternative reinforcers were obtained.

However, there were noticeable between-groups differences in the shape of preference pulses. The preference pulses for IUGR offspring were smaller and fell more sharply in comparison with those of control offspring, even following the first reinforcer (Figure 3, leftmost panels). Log response ratios were smaller for IUGR offspring than for control offspring in 99 of 120 cases $(z=14.7)$, suggesting a reduced effect of individual reinforcers and of sequences of reinforcers on the subsequent behavior of IUGR offspring (sign test, two-tailed, $p<.001$ ). Across all three conditions, log response ratios for IUGR offspring were smaller in 302 of 360 cases (sign test, two-tailed, $p<.001)$.

Figure 4 shows mean total response rates summed across both alternatives as a function of successive reinforcers by group and experimental conditions. Response rates for both groups were superimposable in each condition, and response rates of the IUGR offspring were not significantly different from those of the control group 


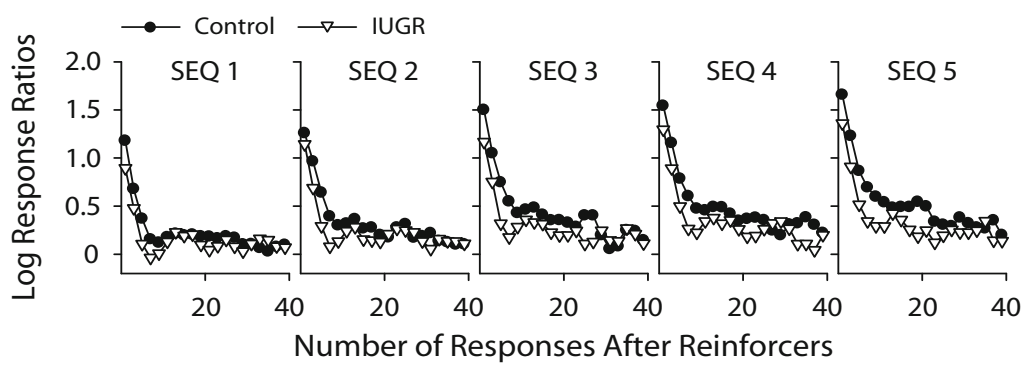

Figure 3. Representative postreinforcer preference pulses from Condition 1, showing changes in preference (log response ratios) as a function of response number (up to 40) for controls (filled circles) and intrauterine growth restricted (IUGR; unfilled triangles) offspring. Sequence 1 (SEQ 1) shows data after a single reinforcer on one alternative; SEQ 2 shows data after two successive reinforcers on one alternative; and so forth.

(repeated measures ANOVA, $p>.05$ ). For both groups, response rates were significantly lower before the first reinforcer (Reinforcer 0) than after the first reinforcer (Reinforcer 1; paired samples $t$ test, $p<.05$ ). After the third reinforcer, response rates did not change significantly for either group (Kendall nonparametric trend test, $p>.05$ ).

Figure 5 shows asymptotic values of sensitivity to reinforcement, calculated by averaging sensitivity values after the last three reinforcers in a component, as a function of body weight. There were no significant correlations between body weights and sensitivity values by groups (Pearson $r, p>.05$ ), either within conditions or overall.

\section{DISCUSSION}

The behavior of all subjects demonstrated that they were able to track changes in reinforcer ratios on the two alternatives, as is evidenced by the fact that sensitivity values increased with increasing numbers of successive reinforcers in components (Figure 2). When the overall reinforcer rate was increased in Condition 2, higher values of sensitivity to reinforcement were obtained in both groups. This increase is the established result, both in steady-state concurrent-schedule procedures (Alsop \& Elliffe, 1988; Elliffe \& Alsop, 1996) and in the frequently changing procedure used in the present study (Davison \& Baum, 2000). However, when the overall reinforcer rate was decreased to the lowest value tested in Condition 3, sensitivity to reinforcement values decreased, but not below the Condition 1 levels. This result, too, is consistent with previous research using this procedure; extended training often results in slightly higher values of sensitivity to reinforcement (e.g., Krägeloh \& Davison, 2003; Landon \& Davison, 2001).

Some investigators have argued that when an independent variable is applied to a litter via the mother, individual pups in a litter do not receive different treatments. Thus, the experimental unit (and the number of subjects, $n$, in a statistical analysis) under assumption would be the number of different dams, rather than the number of different pups - in this case, $n=2$ for the control group, and 3 for the IUGR group. While we will argue against this approach on biological grounds below, if we approach our data in this way, we find that the values of sensitivity to reinforcement values are not significantly different between groups when compared across Reinforcers 1-9 (Figure 2), but that the "asymptotic" values of sensitivity following Reinforcers 7, 8, and 9 remain significantly different between groups in Conditions 1 (2.22 reinforcers $/ \mathrm{min}$ ) and 3 (1.5 reinforcers/min; both $p \mathrm{~s}<.05)$. For the growth curve analysis, the $k$ values were significantly different in Condition 1 ( $t$ test, $p<.05$ ). Thus, this more conservative analysis still largely supports our interpretation of the present data.

However, an alternative statistical argument — considering each individual offspring as the experimental unit, as done here-is based on the biological conditions during fetal development in polytocous species (Festing, 2006). In polytocous mammals, like the rat, significant heterogeneity in phenotypic development is known to exist within each litter. It has been customary to attribute this to prenatal competition for a limited pool of nutrients in the maternal circulation. The fetuses occupying the top (ovarian) position in the uterine horns are on average significantly lighter and

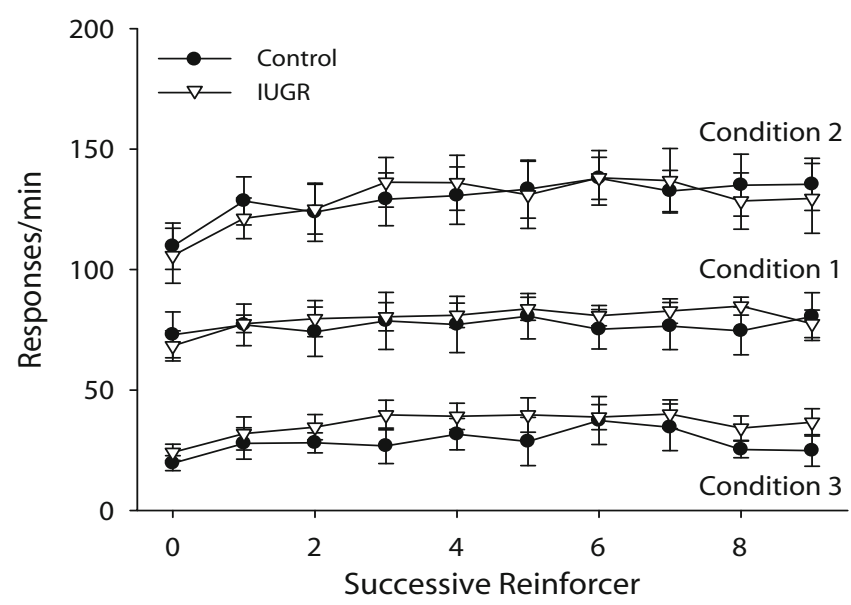

Figure 4. Mean number of responses per minute as a function of successive component reinforcers by conditions for controls (filled circles) and intrauterine growth restricted (IUGR; unfilled triangles) offspring. 


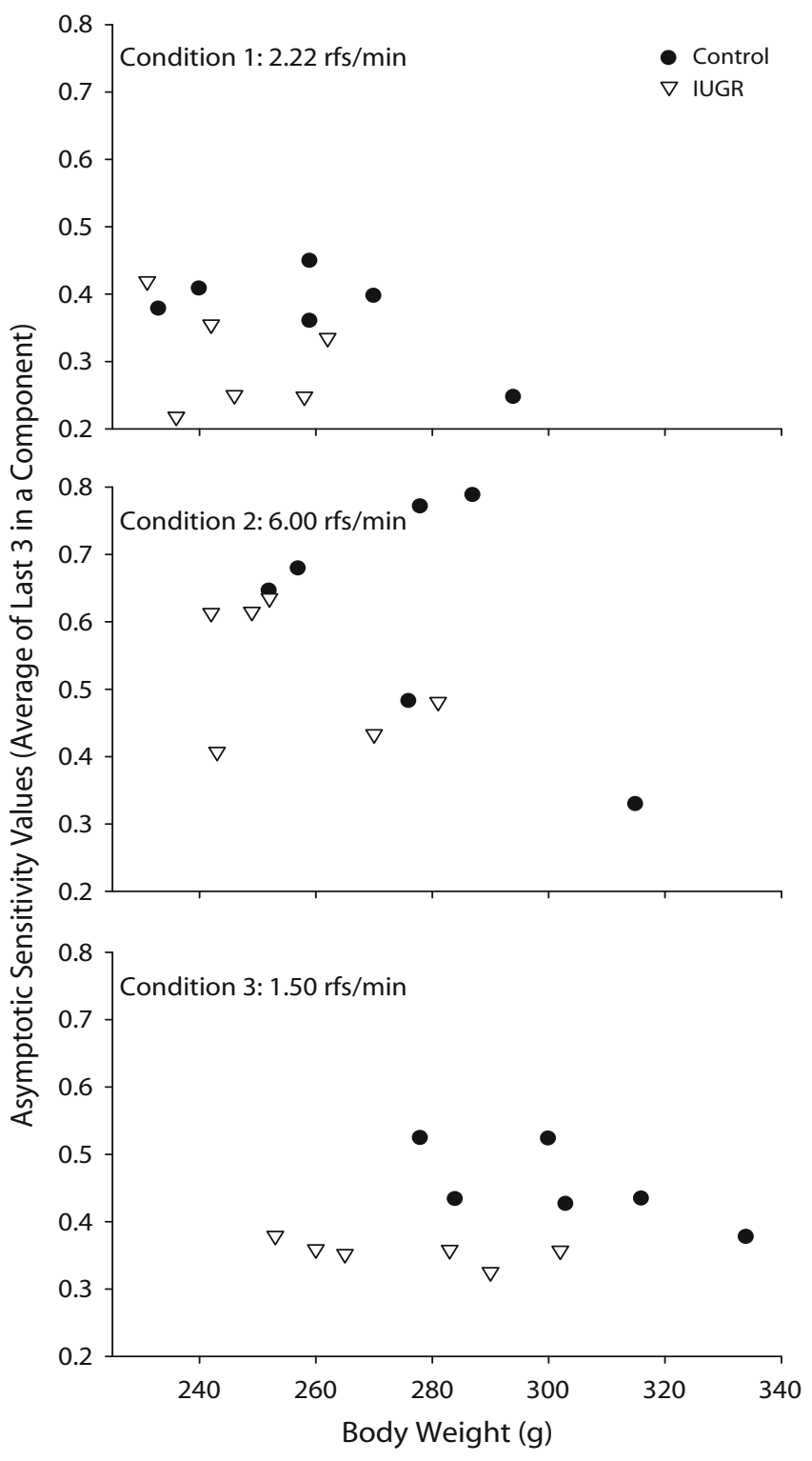

Figure 5. Asymptotic values of sensitivity to reinforcement (average of sensitivity values after Successive Reinforcers 7-9) as a function of body weight in grams by conditions for controls (filled circles) and intrauterine growth restricted (IUGR; unfilled triangles) offspring.

shorter than those located toward the center of the uterus. These conditions are explained by differences in maternal blood supply to the different positions within the uterus. This variation indicates nutritional differences according to location, and hence variations in the nutritional status of the mother will not affect each embryo equally. The statistical argument for using dam numbers rather than individual offspring numbers is thus weakened.

IUGR offspring adapted less well to frequent environmental changes, as was indicated by the fact that sensitivity values were significantly lower than those of control rats (Figure 2). Moreover, underlying this were changes in the form of the response-by-response preference pulses for IUGR offspring in comparison with control offspring (Figure 3). This result shows that IUGR offspring were less likely to stay at the just reinforced alternative than were control offspring. This reduced tendency to follow recent reinforcers undoubtedly contributed to the lower overall values of sensitivity to reinforcement of the IUGR offspring, because the period of responding during the preference pulse contributes substantially to sensitivity values (Baum \& Davison, 2004; Krägeloh \& Davison, 2003). In a recent study, Reed, Paletz, and Newland (2006) made a similar observation of a failure to adapt to changing conditions in a two-lever discrimination procedure resulting from prenatal events. They showed that, in comparison with control rats, rats exposed to methylmercury during gestation tended to exhibit longer perseverative responding to a response lever on which reinforcers were no longer obtainable.

In all experimental conditions, the response rates of the IUGR offspring were similar to those of the control offspring (Figure 4). It is unlikely, therefore, that the observed group differences in sensitivity values resulted from differential motivation or differences in the reinforcing value of food reinforcers. Body weights and sensitivity values were not related in a systematic fashion (Figure 5), either within individual groups or overall. The decreased sensitivity values observed in the IUGR group are therefore not a result of a general effect arising from a lower body weight of the IUGR rats during the time of behavioral testing, but they do appear to be related to the nutritional insult during prenatal development.

The nutritional approach to inducing IUGR used in the present study is a robust and established animal model in which maternal undernutrition has been found to lead to long-term health consequences for the offspring, such as obesity and hypertension, under standard laboratory conditions (Breier, Krechowec, \& Vickers, 2004; Breier et al., 2001). The present results linked this nutritional manipulation during fetal development with reduced behavioral adaptability in later life. Previous research using the same animal model has argued that the difference in the phenotypes of control and IUGR rats is due to early nutritional differences. A possible alternative explanation of these differences could be the (necessary) cross-fostering of the IUGR pups (but not the control pups) onto normal dams to generate a transition from a nutritionally deprived environment before birth to a nutritionally adequate environment after birth. We chose to use the exact experimental approach employed in a number of earlier studies where considerable data are available about the IUGR phenotype (Breier et al., 2001; Krechowec, Vickers, Gertler, \& Breier, 2006; Vickers et al., 2000; Vickers et al., 2001; Woodall et al., 1999; Woodall et al., 1996) so that direct physiological comparisons could be made. Thus, it is possible (though we believe it unlikely) that the learning differences we have documented here arose fully, or partially, from crossfostering. Thus too, future studies will include cross-fostering of all pups, including fostering control pups onto normal dams, to confirm that the behavioral differences 
that we observed in the present study were unequivocally due to undernutrition during fetal development.

The specific neurophysiological mechanisms that underpin these phenotype changes and learning differences remain to be determined, but it is likely that they involve changes in neuroendocrine regulation that are commonly observed after early-life malnutrition and/or structural or functional changes of the hippocampal formation. Protein malnutrition during fetal development has been linked to long-term morphological changes in CA3 hippocampal pyramidal cells, such as decreases of somal size, length of apical dendrites, apical and basal dendritic branching, and spine density (Díaz-Cintra, García-Ruiz, Cordiki, \& Cintra, 1994). Prenatal protein malnutrition can also lead to altered patterns of granule cell neurogenesis in the dentate gyrus (Debassio, Kemper, Tonkiss, \& Galler, 1996) and impairments in establishing long-term potentiation (Bronzino, Austin La France, Morgane, \& Galler, 1996). In the hippocampal formation, alterations in function and structure due to the prenatal nutritional insult appear to be permanent and are not reversed by postnatal nutritional rehabilitation (Dauncey \& Bicknell, 1999).

Gluckman and Hanson (2004) introduced the notion of predictive adaptive responses (PAR). Their theory is that the nutritional environment in early life provides cues for fetal development that prepare the offspring for the nutritional environment that it is likely to encounter after birth. Thus, if the supply of nutrients during fetal development is poor, it is predictable that food supply in the offspring's postnatal environment will also be poor. Development of tissue and metabolic regulation would therefore proceed in such a way as to prepare the fetus for a nutritionally poor environment. A mismatch between the predicted environment and actual environment has been hypothesized as the cause of many metabolic and cardiovascular diseases - for example, when food supply is predicted to be scarce, but turns out to be plentiful after birth

In line with the notion of PAR (Gluckman \& Hanson, 2004; Gluckman, Hanson, Spencer, \& Bateson, 2005), the differences in learning reported in the present study do not necessarily imply learning deficits, but could reflect differences in learning strategies suited for the environment predicted during fetal development. Measures of behavioral adaptation were lower in IUGR offspring (Figure 2), and their behavior tracked recent environmental events more slowly. Moreover, preference pulses were smaller and decreased more rapidly in IUGR offspring than in control offspring (Figure 3), perhaps indicating that the choices of IUGR offspring might be affected by longterm, global events more than are the choices of control offspring. In the present procedure, although there were local fluctuations in the reinforcer ratio on the two alternatives, the overall sessional reinforcer ratio was 1:1 (i.e., in each session, approximately the same number of reinforcers was obtained at each alternative). Thus, if the choices of the IUGR offspring were controlled more by this longer term aggregation, the smaller effects of individual reinforcers and reduced values of sensitivity to reinforcement that we found would be expected.
Epidemiological studies with children that were born small for their gestational age show that these children have slight cognitive deficits that dissipate with time (Richards et al., 2001). The present research provides direct experimental evidence for a link between prenatal nutrition and later learning differences. Since the present study covered 210 days of life in the rat, the present results suggest that differences in learning strategies caused by prenatal nutritional differences are long lasting and, perhaps, permanent. Behavior remains most sensitive to proximal events, but the prospect that the prenatal environment influences adult learning and behavioral adaptation to environmental change has profound public health and societal implications. Even a small but consistent shift in how choices are made or develop throughout the lifespan could compound to result in substantially different outcomes in later life.

To conclude, the data presented here provide sound experimental evidence of prenatal influences on precise measures of learning and behavioral adaptation during adult life in the rat. The IUGR offspring were less adaptable to changes in relative reinforcer rates than were offspring of ad-libitum fed mothers, with values of sensitivity to reinforcement consistently lower after each successive reinforcer. Whether this difference in learning indicates that offspring of undernourished mothers are learning impaired, or whether it reflects different learning strategies suited for different environments, remains to be investigated.

\section{AUTHOR NOTE}

We thank Peter Gluckman and Patrick Bateson for their helpful comments on an earlier version of the manuscript. This work was conducted at the Liggins Institute and was supported by the National Research Centre for Growth and Development and the Health Research Council of New Zealand. We thank Peter Gluckman for his support, Chris Triggs for statistical advice, and Suzanne Logan for helping conduct the experiment. Reprints may be obtained from M. Davison, Department of Psychology, University of Auckland, Private Bag 92019, Auckland, New Zealand (e-mail: m.davison@auckland.ac.nz).

\section{REFERENCES}

Alsop, B., \& Elliffe, D. (1988). Concurrent-schedule performance: Effects of relative and overall reinforcer rate. Journal of the Experimental Analysis of Behavior, 49, 21-36.

BAUM, W. M. (1974). On two types of deviation from the matching law: Bias and undermatching. Journal of the Experimental Analysis of Behavior, 22, 231-242.

BAUM, W. M., \& DAVISON, M. (2004). Choice in a variable environment: Visit patterns in the dynamics of choice. Journal of the Experimental Analysis of Behavior, 81, 85-127.

Breier, B. H., Krechowec, S., \& Vickers, M. H. (2004). Maternal nutrition in pregnancy and adiposity in offspring. In S. C. Langley-Evans (Ed.), Fetal nutrition and adult disease: Programming of chronic disease through fetal exposure to undernutrition (pp. 211-234). Oxford: CABI.

Breier B. H., Vickers, M. H., Ikenasio, B. A., Chan, K. Y., \& Wong, W. P. (2001). Fetal programming of appetite and obesity. Molecular \& Cellular Endocrinology, 185, 73-79.

Bronzino, J. D., Austin la France, R. J., Morgane, P. J., \& Galler, J. R. (1996). Diet-induced alterations in the ontogeny of long-term potentiation. Hippocampus, 6, 109-117.

Dauncey, M. J., \& Bicknell, R. J. (1999). Nutrition and 
neurodevelopment: Mechanisms of developmental dysfunction and disease in later life. Nutrition Research Reviews, 12, 231-253.

DAvison, M., \& BAUM, W. M. (2000). Choice in a variable environment: Every reinforcer counts. Journal of the Experimental Analysis of Behavior, 74, 1-24.

Davison, M., \& BAUM, W. M. (2002). Choice in a variable environment: Effects of blackout duration and extinction between components. Journal of the Experimental Analysis of Behavior, 77, 65-89.

Davison, M., \& McCARThy, D. (1988). The matching law: A research review. Hillsdale, NJ: Erlbaum.

Debassio, W. A., Kemper, T. L., Tonkiss, J., \& Galler, J. R. (1996). Effect of prenatal protein deprivation on postnatal granule cell generation in the hippocampal dentate gyrus. Brain Research Bulletin, 41, 379-383.

Díaz-Cintra, S., García-Ruiz, M., Cordiki, G., \& Cintra, L. (1994). Effects of prenatal malnutrition and postnatal nutritional rehabilitation on CA3 hippocampal pyramidal cells in rats of four ages. Brain Research, 662, 117-126.

Elliffe, D., \& Alsop, B. (1996). Concurrent choice: Effects of overall reinforcer rate and the temporal distribution of reinforcers. Journal of the Experimental Analysis of Behavior, 65, 445-463.

Festing, M. F. W. (2006). Design and statistical methods in studies using animal models of development. ILAR Journal, 47, 5-14.

GlucKman, P. D., \& Hanson, M. A. (2004). Living with the past: Evolution, development, and patterns of disease. Science, 305, 1733-1736.

Gluckman, P. D., Hanson, M. A., Spencer, H. G., \& Bateson, P. (2005). Environmental influences during development and their later consequences for health and disease: Implications for the interpretation of empirical studies. Proceedings of the Royal Society of London: Series $B$, 272, 671-677.

Herrnstein, R. J. (1961). Relative and absolute strength of response as a function of frequency of reinforcement. Journal of the Experimental Analysis of Behavior, 4, 267-272.

KrÄGELOH, C. U., \& DAVISON, M. (2003). Concurrent-schedule performance in transition: Changeover delays and signaled reinforcer ratios. Journal of the Experimental Analysis of Behavior, 79, 87-109.

Krechowec, S. O., Vickers, M., Gertler, A., \& Breier, B. H. (2006). Prenatal influences on leptin sensitivity and susceptibility to dietinduced obesity. Journal of Endocrinology, 189, 355-363.

LANDON, J., \& DAVISON, M. (2001). Reinforcer-ratio variation and its effects on rate of adaptation. Journal of the Experimental Analysis of Behavior, 75, 207-234.

Landon, J., Davison, M., \& Elliffe, D. (2003). Choice in a variable environment: Effects of unequal reinforcer distributions. Journal of the Experimental Analysis of Behavior, 80, 187-204.

LoBB, B., \& Davison, M. C. (1975). Performance in concurrent interval schedules: A systematic replication. Journal of the Experimental Analysis of Behavior, 24, 191-197.

Morgane, P. J., Mokler, D. J., \& Galler, J. R. (2002). Effects of prenatal protein malnutrition on hippocampal function. Neuroscience \& Biobehavioral Reviews, 26, 471-483.
Reed, M. N., Paletz, E. M., \& Newland, M. C. (2006). Gestational exposure to methylmercury and selenium: Effects on a spatial discrimination reversal in adulthood. NeuroToxicology, 27, 721-732.

Richards, M., Hardy, R., KuH, D., \& Wadsworth, M. E. J. (2001). Birth weight and cognitive function in the British 1946 birth cohort: Longitudinal population based study. British Medical Journal, 322 , 199-203.

Richards, M., Hardy, R., KuH, D., \& Wadsworth, M. E. J. (2002). Birth weight, postnatal growth and cognitive function in a national UK birth cohort. International Journal of Epidemiology, 31, 342-348.

StubBs, D. A., \& Pliskoff, S. S. (1969). Concurrent responding with fixed relative rate of reinforcement. Journal of the Experimental Analysis of Behavior, 12, 887-895.

Tonkiss, J., \& Galler, J. R. (1990). Prenatal protein malnutrition and working memory performance in adult rats. Behavioural Brain Research, 40, 95-107.

Tonkiss, J., Galler, J. R., Formica, R. N., Shukitt-Hale, B., \& Timm, R. R. (1990). Fetal protein malnutrition impairs acquisition of a DRL task in adult rats. Physiology \& Behavior, 48, 73-77.

Tonkiss, J., Shultz, P., \& GAller, J. R. (1994). An analysis of spatial navigation in prenatally protein malnourished rats. Physiology \& Behavior, 55, 217-224.

Tonkiss, J., Shultz, P., Shumsky, J. S., \& Galler, J. R. (1997). Development of spatial navigation following prenatal cocaine and malnutrition in rats: Lack of additive effects. Neurotoxicology \& Teratology, 19, 363-372.

Vickers, M. H., Breier, B. H., Cutfield, W. S., Hofman, P. L., \& Gluckman, P. D. (2000). Fetal origins of hyperphagia, obesity and hypertension and its postnatal amplifications by hypercaloric nutrition. American Journal of Physiology: Endocrinology \& Metabolism, 279, E83-E87.

Vickers, M. H., Ikenasio, B. A., \& Breier, B. H. (2001). Insulin-like growth factor-1 treatment reduces hyperphagia, obesity and hypertension in metabolic disorders induced by fetal programming. Endocrinology, 142, 3964-3973.

Woodall, S. M., Breier, B. H., Johnston, B. M., Bassett, N. S. BARNARD, R., \& GLUCKMAN, P. D. (1999). Administration of growth hormone or insulin-like growth factor-I to pregnant rats on a reduced diet throughout pregnancy does not prevent fetal IUGR and elevated blood pressure in adult offspring. Journal of Endocrinology, 163 , 69-77.

Woodall, S. M., Johnston, B. M., Breier, B. H., \& Gluckman, P. D. (1996). Chronic maternal undernutrition in the rat leads to delayed postnatal growth and elevated blood pressure of offspring. Pediatric Research, 40, 438-443.

(Manuscript received August 31, 2006; revision accepted for publication January 10, 2007.) 